\title{
Artículos
}

\section{Evaluación de los costos económicos totales de los desastres naturales: inundación en la ciudad de Sheffield, 2007}

\section{Appraisal of total economic costs of natural disasters: Flood in the City of Sheffield, 2007}

David Mendoza Tinoco*

Alba Verónica Méndez Delgado**

\section{Resumen}

Los desastres naturales representan altos costos que conllevan desequilibrios económicos. Las pérdidas fisicas iniciales generan costos indirectos que amplifican el daño total. Este artículo presenta una metodología basada en el modelo de insumoproducto para evaluar los costos económicos totales de los desastres, y se aplica al caso de las inundaciones de 2007 en Sheffield, Reino Unido. Los resultados sugieren que cada unidad de costo por daños directos, derivados de la destrucción fisica por la inundación, genera un costo adicional por 0.75 unidades en costos indirectos, contabilizado como la pérdida de producción ocasionada por las perturbaciones a lo largo de las cadenas productivas. El análisis brinda información más comprehensiva sobre los efectos de los desastres naturales en la economía, así como sobre los sectores productivos más afectados. Esto podría coadyuvar en una mejor asignación de recursos para la gestión de los riesgos por desastres naturales.

Palabras clave: desastres naturales; inundaciones; modelo insumoproducto; evaluación de desastres; costos directos; costos indirectos.

Clasificación JEL: Q54, Q51, R15.

* Investigador de proyecto, El Colegio de México, A.C., Centro de Estudios Económicos, Programa de Análisis Económico. Dirección postal: Carretera Picacho Ajusco, núm. 20, Col. Ampliación Fuentes del Pedregal, 14110, Ciudad de México. Correo electrónico: dmtinoco@, colmex.mx

** Profesora investigadora de la Universidad Autónoma de Coahuila, Centro de Investigaciones Socioeconómicas. Dirección postal: Unidad Camporredondo, Edificio S, 25280, Saltillo, Coahuila, México. Correo electrónico: albamendez@uadec.edu.mx 


\begin{abstract}
Natural disasters impose high costs that lead to economic imbalances. Losses from initial physical damage generate indirect costs that amplify the total damage. This paper proposes a methodology based on Input Output modelling to evaluate the total economic costs of disasters, and analyses the case of the 2007 floods in Sheffield, $U K$. The results suggest that each unit of cost for direct damages, derived from the physical destruction by the flood, generates an additional cost of 0.75 units in indirect costs, counted as the loss of production caused by the disturbances along the productive chains. The analysis provides more comprehensive information on the effects of natural disasters on the economy, as well as the most affected productive sectors. This could contribute to a better allocation of resources for the risk management of natural disasters.
\end{abstract}

Keywords: natural disasters; floods; input-output model; disaster assessment; direct costs; indirect costs.

JEL Classification: Q54, Q51, R15.

\title{
1. Introducción
}

Los desastres naturales representan elevados costos socioeconómicos en todo el mundo. Algunos de los más devastadores están relacionados con las inundaciones extremas y otros eventos hidrometeorológicos, siendo el Reino Unido uno de los países más afectados por este tipo de fenómenos (Committee of Climate Change, 2016).

La productividad laboral y del capital se ven afectadas negativamente por las inundaciones y crean efectos en cadena que aumentan los costos de las estimaciones de daños iniciales. Es decir, no sólo se ven perjudicados los sectores directamente afectados por el fenómeno, sino que existen efectos indirectos que permean al resto de la economía. Por ejemplo, la inundación ocurrida en Inglaterra durante el verano de 2007 generó una de las mayores emergencias civiles dentro de este país. En la inundación murieron 13 personas y cerca de siete mil fueron rescatadas, 55 mil propiedades fueron afectadas y medio millón de personas experimentaron cortes en los servicios de agua y electricidad (Pitt, 2008). Las regiones más afectadas fueron Yorkshire and The Humber (Y\&H), en el noreste de Inglaterra, donde se concentró $65.5 \%$ de los daños directos nacionales; dentro de esta región, la ciudad de Sheffield sufrió los daños más severos. En el año 2007 Sheffield generó un valor agregado bruto de $£ 8404$ millones, lo cual equivale a $12 \%$ del valor agregado de la región. Es una ciudad que históricamente se ha distinguido por su carácter industrial, siendo una de sus más pujantes ramas 
económicas. Contaba en 2007 con una población de cerca de 540 mil habitantes, de los cuales 248 mil estaban empleados.

Durante las inundaciones del verano de 2007 en Inglaterra, Sheffield sufrió daños materiales por $£ 300$ millones, lo cual representó el $14.3 \%$ de los daños sufridos en la región de Y\&H. Se vieron afectados casi dos mil hogares y más de cuatro mil personas, 64 empresas, además de escuelas y edificios públicos; asimismo, los servicios públicos y la infraestructura se vieron severamente afectados (Ash, Fenn, Daly y Wels, 2008).

Las evaluaciones tradicionales sobre las pérdidas generadas por desastres naturales (en este caso inundaciones) sólo consideran el daño directo en la infraestructura física (Veen, 2004; Cole, 2003; Steenge y Bočkarjova, 2007; Okuyama, 2014; Li et al., 2013). Sin embargo, hay evidencia de efectos en cadena provocados por daños directos, los cuales constituyen una proporción considerable del total de costos socioeconómicos del desastre (Cochrane, 1997; Hallegatte y Przyluski, 2010; Veen, 2004; Santos, Yu, Pagsuyoin y Tan, 2014; Kajitani y Tatano, 2014). Derivado de lo anterior, una eficiente gestión de los riesgos de inundaciones requiere más que una evaluación de pérdidas de productividad laboral y de capital. La evaluación debe también considerar los efectos inducidos durante el proceso de recuperación, los cuales se pueden diseminar a través de los sectores industriales y otras regiones con interdependencias económicas (Hallegate, 2008; Li et al., 2013).

En este artículo se propone aplicar una metodología que permita la medición del impacto económico total producido por un desastre natural (inundación, en este caso), compuesto por sus efectos directos e indirectos en la economía. Esta metodología evalúa los costos directos ocasionados por los daños de la inundación a los factores productivos, a la infraestructura y al capital residencial en la economía afectada, así como los costos económicos indirectos derivados de las pérdidas en la productividad laboral y el capital a lo largo del proceso de recuperación. Se define la pérdida de productividad, de capital o de trabajo como la reducción en el nivel de producción de equilibrio bajo las condiciones dadas antes del desastre natural.

La ventaja de la metodología propuesta es que se basa en el modelo de insumo-producto (IP), con el cual se pueden medir los efectos en cadena (o indirectos), que son el resultado de las interdependencias sectoriales y regionales. La metodología propuesta mejora las evaluaciones de riesgo de las inundaciones existentes, lo que posibilita una mejor preparación y adaptación para eventos futuros.

La metodología empleada se basa en el trabajo expuesto en MendozaTinoco et al. (2017), el cual analiza los costos de la inundación de 2007 en el Reino Unido, pero para la región de Yorkshire and The Humber. En este 
trabajo se aplica una versión ampliada de la metodología al caso específico de una ciudad dentro de la región analizada en el artículo mencionado. Las principales contribuciones de este artículo se constituyen por una extensión metodológica en la cual se añade el concepto de matriz de capital, un elemento dentro de la modelación de insumo-producto (IP) que permite una transición consistente entre la inversión en capital, su acumulación y el correspondiente flujo de producto derivado de ello. Además, el artículo contribuye con una aplicación empírica para las inundaciones que sucedieron en el verano de 2007 en Reino Unido a una escala más pequeña, lo que permite una comparación con los resultados previos sobre una región más amplia y las implicaciones para el caso específico de Sheffield. Para ello fue necesaria la regionalización de la matriz de IP para dicha ciudad, lo cual también contribuye al análisis de la economía de la región.

En la siguiente sección se presenta la revisión de la literatura relevante, relacionada con el análisis basado en la metodología de IP, para la evaluación económica del impacto de los desastres naturales. En la sección 3 se exponen los componentes más importantes de la metodología seguida por MendozaTinoco et al. (2017), así como la incorporación de la matriz de capital a la misma. Los datos necesarios para el análisis se describen en la sección 4. Los resultados principales se presentan en la sección 5. El último apartado presenta una breve discusión a manera de conclusiones.

\section{Investigaciones sobre los efectos de los desastres naturales}

La evaluación del impacto económico de los desastres naturales se ha valido de diversas metodologías que ofrece la economía, tales como los modelos de equilibrio general computable, los modelos econométricos y los modelos de insumo-producto. Dado que los daños físicos de los desastres naturales inducen daños de mayor orden en la economía, debido a las interconexiones económicas de los diferentes agentes y a los desequilibrios ocasionados en los mercados (daños indirectos), las metodologías basadas en el análisis de IP han sido las más utilizadas (Chen et al., 2011, 2015; Okuyama, 2014).

Los modelos de IP se basan en la idea del flujo de bienes intermedios entre los diferentes sectores de producción-que implícitamente representan la tecnología de cada industria-, para la producción de los bienes finales necesarios para satisfacer la demanda final -lo cual implica una economía en equilibrio.

Asimismo, la construcción de la matriz inversa de Leontief, a partir de las transacciones interindustriales contenidas en las tablas de IP, permite 
evaluar los efectos indirectos a lo largo de la cadena de valor. Otra ventaja del análisis es que la calibración necesaria de los parámetros es menor que la requerida por otras metodologías. También es posible la regionalización de las tablas nacionales de IP, lo que posibilita un análisis regional. Estas características permiten estimar las pérdidas de orden superior, o indirectas. Sin embargo, el modelo IP estándar presenta algunas limitaciones: es un modelo estático que sólo responde a cambios en la demanda, utiliza funciones de producción de proporciones fijas, los precios son fijos, y no se consideran la sustitución de insumos ni de importaciones (Cochrane, 2004; Cole, 2003; Greenberg et al., 2007; Li et al., 2013; Okuyama, 2014; Rose, 2004).

\subsection{Marco analítico}

Esta sección expone la investigación basada en el análisis de IP para la evaluación del impacto de los desastres naturales.

Una de las afectaciones a la economía ocasionadas por los desastres naturales es el rompimiento del equilibrio económico, ya que de éste se derivan interrupciones en los procesos productivos que generan costos indirectos. Para considerar dichos desequilibrios dentro del análisis de IP, Steenge y Bočkarjova (2007) presentan el concepto de la matriz de contabilidad de daños del evento (EAM, por sus siglas en inglés). Se trata de un componente matemático (una matriz diagonal) cuyos elementos diagonales expresan la proporción dañada de la capacidad productiva de cada sector. Los desequilibrios y los posibles cuellos de botella después de un choque se derivan de la información en la EAM, y la trayectoria de recuperación se traza a partir de este punto.

Por su parte, Hallegatte (2008) incorpora al modelo la dimensión temporal para analizar la trayectoria de recuperación. El modelo de IP adaptativo regional (MIPAR) considera tanto los cuellos de botella causados por los daños a la capacidad productiva industrial, como el comportamiento adaptativo de los consumidores y productores frente a los desequilibrios. Cabe mencionar que el modelo no considera los cuellos de botella resultantes de las restricciones en la capacidad productiva del trabajo, ni tampoco el daño al capital residencial (Li et al., 2013).

A partir del MIPAR, Li et al. (2013) proponen un modelo para analizar la recuperación de una economía en desequilibrio. En este modelo se incorporan las restricciones a la producción, no sólo las causadas por daños industriales, sino también considerando las reducciones en la productividad como resultado del daño a la fuerza laboral. El modelo también toma en 
cuenta el daño residencial, que interactúa con el proceso de reconstrucción durante la competencia por los recursos disponibles y afecta la recuperación de la capacidad laboral.

Por su parte, Dietzenbacher y Lahr (2013) aplican el método de extracciones hipotéticas al análisis de la evaluación de impacto. La principal contribución de este enfoque es que considera consistentemente los efectos hacia adelante (en la cadena productiva) de un choque en alguno de los sectores productivos.

A su vez, Koks, Bockarjova, de Moel y Aerts (2014) proponen usar una función Cobb-Douglas para estimar los daños directos de mano de obra y restricciones de capital, mientras que los daños indirectos incurridos durante el proceso de recuperación se calculan mediante un MIPAR.

Recientemente, Mendoza-Tinoco et al. (2017) desarrollaron lo que llaman la huella de inundación, la cual es un marco analítico de evaluación de los daños tanto en la región inundada como en los sistemas económicos interconectados a mayor escala. Ellos aplican la metodología para la región que contiene a la ciudad de Sheffield, que en este artículo se analiza.

Una crítica generalizada a los métodos de impactos, y de lo cual adolecen las metodologías mencionadas, es que no existe un sistema claro y consistente que explique la relación entre la restauración del capital y la recuperación de la capacidad productiva.

Este artículo sigue la metodología desarrollada en Mendoza-Tinoco et al. (2017), e incorpora el concepto de la matriz de capital para dar consistencia metodológica a la transición entre una variable de stock (el capital industrial) y una variable de flujo (la producción) en el proceso de recuperación de la economía. Se aplicó la metodología para medir el impacto económico total que fue directa e indirectamente causado por las inundaciones del verano de 2007 en Sheffield.

En este modelo se combinan las ventajas de los modelos existentes utilizados en el análisis de riesgo de desastres, incluyendo el análisis de los daños al capital de los sectores industriales, las afectaciones a la fuerza laboral, los desequilibrios económicos posteriores a los desastres y los cuellos de botella en la oferta. Para modelar el proceso de recuperación, se propone la asignación de recursos mediante un esquema de racionamiento para satisfacer la restauración del capital industrial y los daños a los hogares. Y se incorpora la matriz de capital para modelar la recuperación de la capacidad productiva, debido a la inversión en bienes de capital para reestablecer el capital productivo dañado. Las posibilidades de cambios en la demanda final también se explican a través de la modelación del comportamiento adaptativo de los consumidores. 


\subsection{Aplicaciones de evaluación de impacto}

La evaluación de impacto por medio del análisis de IP y otras metodologías relacionadas ha resultado de gran ayuda para la estimación de los costos económicos ocasionados por grandes desastres naturales.

Por ejemplo, Hallegate (2008) estima los costos que el huracán Katrina ocasionó en el estado estadounidense de Luisiana. Por medio de un MIPAR calcula que los costos directos, estimados en 107 billones de dólares, ocasionaron daños indirectos por un valor de 42 billones de dólares. Esto indica que por cada dólar de daños a la infraestructura física, se perdió la producción de bienes con valor de 0.40 dólares.

Otro fenómeno natural que causó estragos en el ámbito nacional es el gran terremoto y tsunami de 2011 en el este de Japón. Kajitani y Tatano (2014) estiman la tasa de pérdida de capacidad productiva para varios sectores económicos, principalmente los manufactureros. Basado también en el análisis de IP, la metodología es capaz de proveer información sobre la escala de los trastornos en la capacidad productiva de los sectores económicos. Los resultados estiman que los daños directos sólo representan $40 \%$ del total de estragos económicos, siendo la mayoría atribuidos a las perturbaciones en la capacidad productiva. También se muestra una correlación positiva y significativa entre los resultados y los niveles de producción reportados por el índice de producción industrial japonés.

\section{Metodología}

Este artículo aplica en su mayoría la metodología desarrollada en MendozaTinoco et al. (2017), por lo que se sugiere remitirse a dicho artículo para una exposición más detallada del modelo.

En esta sección se presentan las principales ecuaciones de la metodología y se incorpora el concepto de matriz de capital

La metodología se basa en el modelo de IP. ${ }^{1}$ Las tablas de IP presentan las transacciones interindustriales de toda la economía en una matriz lineal. En la notación matemática se presenta como:

${ }^{1}$ Nota respecto a los símbolos y fórmulas: las matrices están representadas por letras mayúsculas en cursiva en negrita $(\boldsymbol{X})$, vectores en cursiva negrita $(\boldsymbol{x})$ y escalares en minúscula cursiva $(x)$. Los vectores son de columna; los vectores de fila se obtienen transponiendo el vector $\left(x^{\prime}\right)$. Una conversión de un vector a una matriz diagonal se expresa como una letra minúscula con un circunflejo $(\hat{x})$. Los operadores '.*' y './' se utilizan para expresar la multiplicación elemento por elemento y la división elemento por elemento de dos vectores, respectivamente. 


$$
\boldsymbol{x}=\boldsymbol{A x}+\boldsymbol{f}
$$

donde $\boldsymbol{x}$ es un vector de dimensión $1 x n$ ( $n$ es el número de sectores) que representa la producción total de cada sector. ${ }^{2} \boldsymbol{A} \boldsymbol{x}$ representa el vector de demanda intermedio, donde cada elemento de la matriz $\boldsymbol{A}\left(\left[a_{i j}\right]\right)$ es el coeficiente técnico que indica la cantidad del producto $i$ necesario para producir una unidad del producto $j$. Por último, $\boldsymbol{f}$ indica el vector de demanda final de los productos.

Para considerar otro de los principales factores productivos, el trabajo, se hace uso de la ecuación básica desarrollada por Steenge y Bockarjova (2007) (véase la Ecuación 2), que contiene todas las transacciones intermedias de bienes y requerimientos de mano de obra por sector. En este punto se considera que la economía está en equilibrio con pleno empleo de los factores productivos:

$$
\left[\begin{array}{cc}
A & \mathbf{f} / l \\
\mathbf{l}^{\prime} & 0
\end{array}\right]\left(\begin{array}{l}
\mathbf{x} \\
l
\end{array}\right)=\left(\begin{array}{l}
\mathbf{x} \\
l
\end{array}\right)
$$

Después de un desastre, las fuerzas del mercado se desequilibran, lo que genera brechas entre la oferta-ocasionadas por las perturbaciones a la capacidad productiva de la industria y el trabajo- y la demanda -provocadas por cambios de comportamiento y los requerimientos de bienes de reconstrucción-. Entonces, la capacidad total de producción de la economía, después de considerar las restricciones del capital industrial y la fuerza de trabajo, es igual al mínimo de ellas:

$$
\mathbf{x}_{\mathrm{tp}}^{\mathrm{t}}=\min \left\{\mathbf{x}_{\text {cap }}^{\mathrm{t}}, \mathbf{x}_{1}^{\mathrm{t}}\right\}
$$

donde $\mathbf{x}_{c a p}^{\mathbf{t}}$ es la capacidad productiva del capital en el tiempo $t$, y $\mathbf{x}_{l}^{\mathbf{t}}$ es la capacidad productiva del trabajo en el tiempo $t$ de la recuperación.

Asimismo, la demanda final total de cada periodo a lo largo de la recuperación, para cada sector, considera las necesidades de insumos de dicha industria, más la demanda final que incluye los bienes de capital necesarios para la reconstrucción (Ecuación 4). ${ }^{3}$

$$
\mathrm{x}_{\mathrm{td}}^{\mathrm{t}}(\mathrm{i})=\sum_{\mathrm{j}=1}^{\mathrm{n}} a(i, j) o^{t}(\mathrm{j})+\mathrm{f}_{r e c}^{\mathrm{t}}(\mathrm{i})
$$
precio.

${ }^{2}$ En el modelo se supone que cada sector produce un producto uniforme con un único

${ }^{3}$ Los índices en paréntesis indican el sector industrial. 
donde $o^{t}(j)$ representa los requerimientos de insumos intermedios de la industria $i$ para el resto de los sectores. Y $f_{\text {rec }}^{t}(i)$ representa la demanda final que incorpora la de bienes para la restauración de los daños.

Para el proceso de recuperación económica, se incorporó a la metodología el concepto de matriz de capital, que permite una transformación metodológicamente consistente de la inversión de capital a la capacidad productiva.

\subsection{Proceso de recuperación después de un desastre}

Una economía se considera recuperada una vez que las capacidades laborales y de producción industrial están en equilibrio, donde la demanda total y la producción recobran el nivel anterior al desastre. La forma de utilizar los recursos restantes, para alcanzar las condiciones anteriores al siniestro, se modelan siguiendo un esquema de racionamiento.

El primer paso es determinar la capacidad de producción disponible en cada periodo posterior al desastre. En el contexto de las funciones de producción de Leontief, la capacidad productiva está determinada por el mínimo de cada factor productivo, capital y trabajo, como se muestra a continuación:

$$
\boldsymbol{x}_{t p}^{t}=\min \left\{\boldsymbol{x}_{c a p}^{t}, \boldsymbol{x}_{l}^{t}\right\}
$$

En segundo lugar, el nivel de la capacidad de producción restringida se compara con la demanda total para determinar la estrategia de asignación de los recursos restantes y para la planificación de la reconstrucción. Las reglas de este proceso constituyen lo que se llama el esquema de racionamiento, descrito a continuación.

\subsubsection{Esquema de racionamiento}

El proceso de recuperación requiere la asignación de los recursos restantes para satisfacer las necesidades de la sociedad durante las consecuencias del desastre. En este trabajo se utilizó un esquema de racionamiento de priorización proporcional, que asigna primero la producción restante entre la demanda interindustrial $\left(\boldsymbol{A} \boldsymbol{x}_{t p}^{t}\right)$ y luego se enfoca en las categorías de demanda final. ${ }^{4}$

${ }^{4}$ Se supone que la productividad de cualquiera de los factores productivos no cambia durante el proceso de recuperación, como es el caso de las funciones de producción de Leontief. También se hace el supuesto de que el desastre ocurre justo después del tiempo $t=0$ y que el proceso de recuperación comienza en $t=1$. 
Al calcular las posibilidades productivas del periodo siguiente, la producción real se compara primero con la demanda interindustrial. Definiendo $o_{i}^{t}=\sum_{j} A_{i j} x_{t p(j)}^{t}$ como la producción requerida en la industria para satisfacer la demanda intermedia de las otras industrias, dos posibles escenarios pueden surgir después del desastre (Hallegatte, 2008).

El primer escenario ocurre si $x_{t p(i)}^{t}<o_{i}^{t}$, en cuyo caso la producción de la industria $i$ en el tiempo $t$ en la situación post-desastre $\left(x_{t p(i)}^{t}\right)$ no puede satisfacer las demandas intermedias de otras industrias. Esta situación constituye un cuello de botella en la cadena de producción, donde la producción en la industria $j$ se ve limitada por $\frac{x_{t p(i)}^{t}}{o_{i}^{t}} x_{t p(j)}^{t}$, donde $\frac{x_{t p(i)}^{t}}{o_{i}^{t}}$ es la proporción de la producción restringida en la industria $j\left(x_{t p(j)}^{t}\right)$.

Este proceso ocurre para cada industria; después debe considerarse el hecho de que las industrias que producen menos también demandarán menos, afectando y reduciendo la producción de otras industrias. La iteración de este proceso continúa hasta que la capacidad de producción puede satisfacer la demanda intermedia adaptada y una fracción de la producción restante se libera para satisfacer parte de la demanda final y de reconstrucción, y así aumentar la capacidad productiva del siguiente periodo. Esta situación conduce a un equilibrio parcial, donde se define $\boldsymbol{A} \boldsymbol{x}_{t p}^{t}$ como el nivel de la demanda intermedia adaptada, el asterisco en $\boldsymbol{x}_{t p}^{t^{*}}$ representa la capacidad de producción adaptada que proporciona el equilibrio parcial y es menor que la capacidad de producción real ( $\left.\boldsymbol{x}_{t p}^{t}\right)$ de la Ecuación 11 .

Este proceso continúa hasta que la producción total disponible en cada momento $\left(x_{t p(i)}^{t}\right)$ pueda satisfacer la demanda intermedia en el tiempo $t, o_{i}^{t}$.

El segundo escenario se produce cuando $x_{t p(i)}^{t}>o_{i}^{t}$. Entonces, la demanda intermedia se puede satisfacer sin afectar la producción de otras industrias.

Cabe mencionar que el primer escenario sólo ocurre cuando el choque a la economía ha sido de tal magnitud que alguna(s) de las empresas no son capaces de satisfacer siquiera la demanda intermedia. Si es así, se modela primeramente la recuperación de la economía para satisfacer la demanda intermedia, después de lo cual sucede el segundo escenario. En caso de que la economía pueda satisfacer la demanda intermedia después del desastre, sólo se modela el segundo escenario.

En ambos casos, la producción restante después de satisfacer la demanda intermedia se asigna proporcionalmente a la demanda de recuperación y a otras categorías de demanda final. 
Adicionalmente se supone que parte de la demanda final insatisfecha está cubierta por importaciones, algunas de las cuales contribuyen a la recuperación cuando se asignan a la demanda de reconstrucción.

\subsubsection{Matriz de capital}

Esta sección describe la incorporación de la matriz de capital al marco analítico de la evaluación del impacto económico, para lograr una transformación metodológicamente consistente de la inversión de capital a la capacidad productiva. La matriz de capital se utiliza tradicionalmente en el análisis IP para simular el crecimiento económico mediante la acumulación de capital. El uso de dicha matriz en el análisis de impacto económico de los desastres naturales fue propuesta originalmente por Albert Steenge (Triple E Consulting, 2014), quien considera la inversión en restauración como una variable exógena que permite planificar la recuperación.

La matriz de capital se adapta dentro del marco analítico que hemos seguido, donde la inversión en reconstrucción se asigna de acuerdo con la proporción de la demanda de reconstrucción, en relación con las otras categorías de demanda final. Como se mencionó en el esquema de racionamiento, se supone que la producción superviviente se asigna a las diferentes categorías de demanda final una vez satisfecha la demanda intermedia.

Una matriz de capital, $\mathbf{K}$, es una matriz cuadrada donde cada elemento $[k(i, j)]$ denota la cantidad de bienes de capital producidos por el sector $i$ para aumentar la capacidad de producción del sector $j$ en una unidad. Es decir, los elementos de la columna $j$ representan la "receta" de la maquinaria, equipo y otros bienes de capital necesarios para que el sector $j$ incremente su productividad en una unidad, manteniendo constante el nivel de insumos y tecnología (Miller y Blair, 2009).

Debe recordarse que el proceso de recuperación requiere la reparación y/o reemplazo del stock de capital dañado y residencial. Durante este proceso, la capacidad de producción aumenta a través de la producción local y las importaciones asignadas a la inversión en reconstrucción. Nótese que la reconstrucción del capital residencial es a través del consumo de productos finales a los sectores de reconstrucción.

La inversión de capital para la reconstrucción, $\mathbf{K} * \Delta \mathbf{x}_{\text {cap }}^{\mathrm{t}}$, se calcula como la participación de la demanda de reconstrucción entre la demanda final total, multiplicada por la producción restante después de satisfacer la demanda intermedia, $\left(\mathbf{x}_{\mathrm{tp}}^{\mathrm{t}}-\mathbf{A} \mathbf{x}_{\mathrm{td}}^{\mathrm{t}}\right)$, como se muestra en la Ecuación 6: 


$$
\mathbf{K} * \Delta \mathbf{x}_{\text {cap }}^{\mathrm{t}}=\left(\mathbf{x}_{\mathrm{tp}}^{\mathrm{t}}-\mathbf{A} \mathbf{x}_{\mathrm{td}}^{\mathrm{t}}\right) *\left(\mathbf{f}_{\text {cap }}^{\mathrm{t}} / /\left(\sum_{k} \mathbf{f}_{\mathrm{k}}^{0}+\mathbf{f}_{\mathrm{rec}}^{\mathrm{t}}\right)\right)
$$

Cabe señalar que, en este caso, la inversión en restauración de capital industrial implica tanto los requerimientos técnicos de capital, por industria, desglosados en la matriz de capital $\mathbf{K}$, así como la cantidad de capacidad productiva que se añade al siguiente periodo $\left(\Delta \mathbf{x}_{\text {cap }}^{\mathrm{t}}\right)$.

Asimismo, se puede expresar la participación de las importaciones que se invierten en la reconstrucción del capital, para estimar su contribución al aumento de la capacidad de producción durante el proceso de reconstrucción. Una vez que el monto de las importaciones designadas para la inversión de capital se determina, como en la Ecuación 7, se puede obtener fácilmente la capacidad productiva que ha sido restaurada mediante importaciones $\left(\Delta \mathbf{x}_{\mathrm{m}}^{\mathrm{t}}\right)$.

$$
\mathbf{K} * \Delta \mathbf{x}_{\mathrm{m}}^{\mathrm{t}}=\mathbf{m}^{\mathrm{t} *}\left[\mathbf{f}_{\text {cap }}^{\mathrm{t}} \cdot /\left(\sum_{k} \mathbf{f}_{\mathrm{k}}^{0}+\mathbf{f}_{\mathrm{rec}}^{\mathrm{t}}\right)\right]
$$

Debe recordarse que la suma $\sum_{k} \mathbf{f}_{\mathrm{k}}^{0}$ representa la demanda final total de las categorías, $k$ : hogares, gobierno, capital, exportaciones.

Entonces, la inversión total en la restauración del capital de cada periodo es:

$$
\mathbf{K} * \Delta \mathbf{x}^{\mathrm{t}}=\mathbf{K} *\left(\Delta \mathbf{x}_{\mathrm{tp}}^{\mathrm{t}}+\Delta \mathbf{x}_{\mathrm{m}}^{\mathrm{t}}\right)
$$

La multiplicación por la inversa de la matriz de capital proporciona la capacidad productiva industrial que se añade para el siguiente periodo, $\Delta \mathbf{x}^{\mathrm{t}}=\Delta \mathbf{x}_{\mathrm{tp}}^{\mathrm{t}}+\Delta \mathbf{x}_{\mathrm{m}}^{\mathrm{t}}$.

Así, para el siguiente periodo, las posibilidades de producción de la capacidad industrial están dadas por la siguiente expresión:

$$
\mathbf{x}_{\text {cap }}^{\mathrm{t}+1}=\mathbf{x}_{\text {cap }}^{\mathrm{t}}+\Delta \mathbf{x}^{\mathrm{t}}
$$

Esto permite reformular la función del vector $\mathbf{f}_{\mathrm{rec}}^{\mathrm{t}}$ en términos de una matriz de capital de Leontief $(\mathbf{K})$. Al sustituir el término $\Delta \mathbf{x}^{\mathrm{t}}$ en la Ecuación 9, en términos de la matriz de capital, se obtiene la demanda total que la economía necesita en cada periodo durante el proceso de recuperación:

$$
\mathbf{x}_{\mathrm{td}}^{\mathrm{t}}=\mathbf{A} \mathbf{x}_{\mathrm{td}}^{\mathrm{t}}+\sum_{k} \mathbf{f}_{\mathrm{k}}^{0}+\mathbf{f}_{\mathrm{hd}}^{\mathrm{t}}+\mathbf{K} \Delta \mathbf{x}^{\mathrm{t}}
$$


Una nueva iteración de este proceso comienza nuevamente y prosigue hasta que la demanda total y la producción total se encuentren en equilibrio y en el mismo nivel que antes del desastre.

\subsection{Impacto económico total}

Por último, el impacto económico total del evento (tec) es la suma de los costos directos $\left(\mathbf{v} \mathbf{a}_{\text {dir }}\right)$ y los costos indirectos $\left(\mathbf{v a}_{\text {ind }}\right)$ generados durante cada periodo del proceso de recuperación, como se expresa en la Ecuación 11. Los costos se miden en términos de valor agregado, que en el caso de los daños directos es igual al costo de reposición, a precios de mercado. Esto constituye la demanda total de recuperación, $\boldsymbol{f}_{\text {rec }}^{0}$. Por otro lado, el costo indirecto se calcula como la acumulación de las diferencias entre el nivel de producción antes del desastre $\left(\mathbf{x}^{0}\right)$ y la producción restringida después del desastre en cada periodo $\left(\mathbf{x}_{\mathrm{tp}}^{\mathrm{t}}\right)$, lo cual es igual al término $\left(T\left(\mathbf{x}^{0}\right)-\sum_{\mathrm{t}} \mathbf{x}_{\mathrm{tp}}^{\mathrm{t}}\right)$, donde $T$ es el tiempo estimado para la recuperación de la economía.

$$
\mathbf{t e c}=\left(\mathbf{v} \mathbf{a}_{\text {dir }}+\mathbf{v} \mathbf{a}_{\text {ind }}\right)=\mathbf{f}_{\text {rec }}^{0}+\left(T\left(\mathbf{x}^{0}\right)-\sum_{\mathrm{t}} \mathbf{x}_{\mathrm{tp}}^{\mathrm{t}}\right)
$$

\section{Recopilación y codificación de datos}

La metodología propuesta requiere dos conjuntos de datos: los económicos sobre la región afectada, y la información referente a los daños ocasionados por el desastre. Todos los valores son para el año 2007, y cuando son monetarios están en millones de libras ( $£$ millones) a precios de 2009. Se utiliza una escala de tiempo mensual para el análisis temporal, y la desagregación sectorial es de 46 sectores económicos (véase la desagregación sectorial en el VDE del Apéndice A).

\subsection{Datos económicos}

Los datos económicos incluyen información sobre el stock de capital, la demanda final, el empleo y las transacciones interindustriales. Toda la información se encuentra a nivel regional y, cuando está disponible, se obtiene a partir de datos oficiales. De lo contrario, se lleva a cabo una regionalización a partir de métodos estadísticos. 
Los datos sobre el stock de capital sólo están disponibles a escala nacional. La regionalización consistió en obtener la productividad de cada sector a nivel nacional y luego se ponderó por la producción regional, asumiendo la misma productividad que la media nacional en cada sector. El capital de vivienda regional es la proporción de vivienda en la región, multiplicada por el capital de vivienda nacional.

Los datos para las categorías para la demanda final, es decir, los hogares, el gobierno, el capital, las importaciones y las exportaciones, se obtuvieron del modelo dinámico multisectorial británico (MDM, por sus siglas en inglés) de Cambridge Econometrics Ltd. Éste es un modelo macroeconómico para el análisis de las relaciones de la economía, el medio ambiente y consumo energético, para doce regiones en el Reino Unido. Los datos utilizados fueron para la región de $\mathrm{Y} \& \mathrm{H}$, desagregados en 46 sectores industriales. Posteriormente se llevó a cabo la regionalización para la ciudad de Sheffield.

Los datos sobre el empleo suelen estar disponibles a una escala regional muy detallada; éstos se obtuvieron directamente de los datos oficiales y sirvieron para la regionalización de las demás categorías. Sin embargo, la desagregación sectorial no fue consistente con el resto de los datos. Para hacer coincidir la desagregación sectorial de los datos del empleo con la desagregación de 46 sectores, se siguió una distribución ponderada basada tanto en el empleo nacional como en los datos sobre el valor agregado obtenidos del MDM.

Las tablas de IP regionales fueron construidas a partir de las tablas nacionales mediante el método de coeficientes de localización aumentados de Flegg (AFLQ, por sus siglas en inglés) (véase el Apéndice B).

\subsection{Información sobre daños del desastre}

Idealmente, los datos sobre los daños ocasionados por los desastres comprenden información de daños al capital industrial, al capital residencial y a la infraestructura, así como trastornos de la capacidad laboral y cambios en la demanda final.

La principal fuente de información para los datos de desastres es la Agencia de Medio Ambiente del Reino Unido (EA, por sus siglas en inglés), y la información para el evento analizado se describe en el informe "Economic impacts of flood risk on YyH. Cost of 2007 floods" (Ash et al., 2008).

Para los daños al capital industrial, el informe indica un costo total de $£ 138$ millones para locales comerciales, inventarios, equipo, etc. Además, los $£ 148$ millones de daños a la infraestructura se distribuyeron entre los 
sectores de transporte; servicios de tecnologías de la información; electricidad y gas; agua, alcantarillado y residuos; administración pública y defensa (PAD, por sus siglas en inglés), educación, y salud. Dado que la desagregación sectorial de los daños fue para 46 categorías, se asignó el valor del daño a cada sector industrial a través de una distribución ponderada basada en la participación del sector en la economía regional. Estos datos se compararon con el capital industrial para determinar la proporción de la capacidad productiva afectada; es decir, se estima el daño productivo a cada sector como la proporción de los daños materiales por sector, respecto al valor del stock de capital del mismo. Estos valores constituyen los valores del VDE (véase el Apéndice A). Para los sectores manufactureros la proporción de daños al capital industrial representa $0.7 \%$, mientras que para los sectores relacionados a la infraestructura representa $1.4 \%$ para los sectores relacionados a la provisión de energía (gas y electricidad) y de agua; y $1.7 \%$ para la industria del transporte. Mientras que para los sectores de servicios relacionados con las telecomunicaciones, la administración pública, y los servicios recreacionales, los daños al capital representaron 1.4, 1.1 y 1\%, respectivamente.

Respecto a los daños residenciales, 1793 casas resultaron inundadas, que representan el $0.4 \%$ del total de viviendas en la región. El Departamento de Medio Ambiente del Reino Unido estimó en $£ 12.7$ millones el total de daños a los hogares.

Las afectaciones a la fuerza laboral se derivaron del número de viviendas inundadas multiplicado por el número medio de trabajadores por hogar, ya que no se cuenta con datos duros al respecto. Además, los retrasos en los traslados se relacionaron proporcionalmente con los daños en los sectores del transporte. Entonces, se calculó que la mano de obra afectada fue igual a un décimo de la proporción de daños al capital del sector transporte, y que $0.5 \%$ de la población sufrió retrasos de tres horas en sus desplazamientos.

Por último, dado que la información sobre los cambios en la demanda final es muy escasa, seguimos un análisis de sensibilidad sobre los diferentes niveles de reducción de los productos no básicos. Los valores para el análisis consideran una disminución igual al valor mínimo de daño al capital productivo entre los sectores industriales, para las industrias no básicas. Se consideró un tiempo de recuperación de 10 meses con crecimiento lineal. 


\section{Resultados}

\subsection{Costos económicos totales para la ciudad de Sheffield}

Sheffield se encuentra localizada en la región $Y \& H$, que se ubica al noroeste del Reino Unido. El valor agregado bruto (VAB) anual en 2007 superó los $£ 8404$ millones (a precios de 2009), lo que representa alrededor del 0.73\% del valor agregado total del Reino Unido para ese año. Asimismo, hay cerca de 248 mil empleados en la región, que constituyen alrededor del $0.8 \%$ de la fuerza laboral total del Reino Unido.

Según el análisis con la metodología propuesta, la economía de Sheffield tarda al menos 17 meses en volver a su situación previa al desastre, después de las inundaciones en el verano de 2007 en el Reino Unido (Figura 1). Para este análisis de impacto, la recuperación significa que la economía se encuentra nuevamente en equilibrio y que el nivel de producción en este equilibrio es el mismo que en la situación previa al desastre. Los daños suponen una pérdida económica total de $£ 524$ millones, lo que equivale al $5.7 \%$ del VAB regional en ese año.

\section{Figura 1}

Distribución del daño por categoría (£ millones)

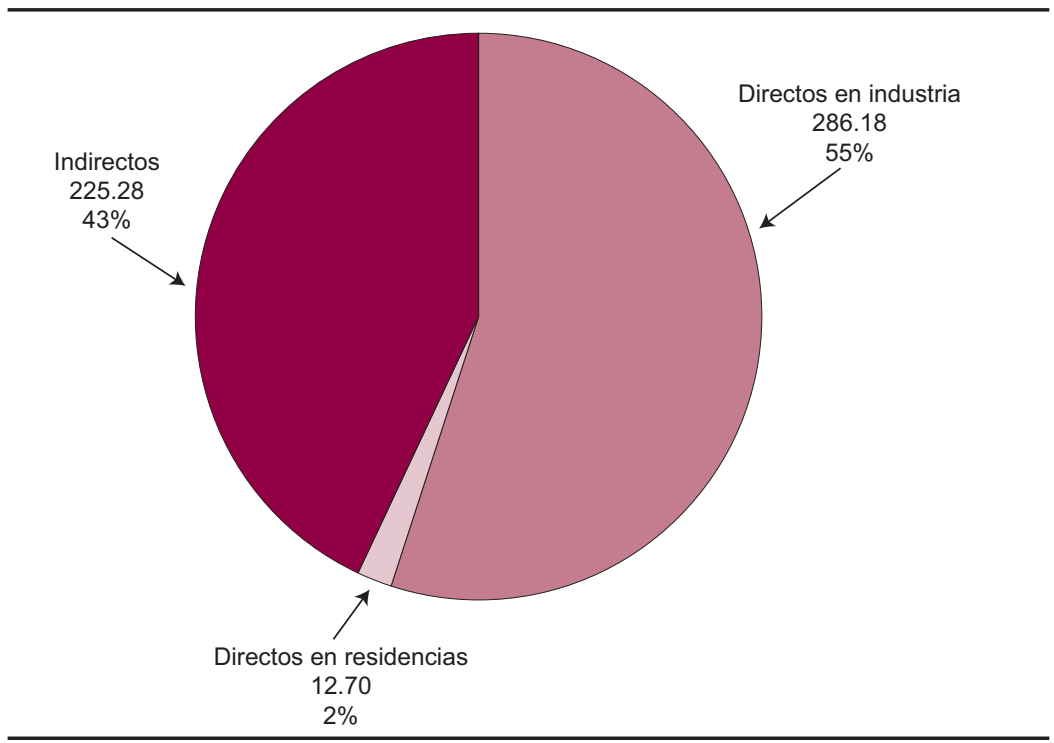

Fuente: Elaboración propia. 
Al diferenciar la pérdida económica directa de la pérdida económica indirecta, la Figura 2 compara las proporciones de cada categoría. Las pérdidas económicas directas (incluidos los daños a la infraestructura industrial y residencial) representan 3.2\% del VAB anual (casi $£ 300$ millones), de las cuales la mayoría corresponde a daños industriales e infraestructurales (55\%). La pérdida económica indirecta -que es la suma de todos los flujos de producción no realizados debido a las interrupciones de la productividad y la demanda-representa un $2.4 \%$ adicional del VAB de la ciudad (cerca de $£ 225$ millones). Esto representa 43\% del impacto económico total.

\subsection{Recuperación económica}

La presente sección describe la trayectoria de las variables económicas involucradas en el proceso de recuperación.

La Figura 2.a representa el daño acumulado durante el proceso de recuperación. El área en púrpura, que indica la distancia entre la demanda final satisfecha por la producción disponible en el periodo y el nivel anterior al desastre, representa el daño indirecto total durante el proceso de recuperación. Cabe señalar que el choque inicial representa una disminución de $11 \%$ de la capacidad productiva. La forma de la curva muestra una recuperación rápida al principio, especialmente en los primeros cinco meses, tiempo en que la economía ha recuperado aproximadamente el $90 \%$ de la capacidad productiva dañada. Cabe mencionar que la forma de curva de recuperación está influenciada por el esquema de racionamiento elegido para el modelo, donde la demanda interindustrial y la demanda de recuperación se priorizan sobre el resto de la demanda final.

La Figura 2.b muestra el proceso de recuperación de la capacidad productiva, incluyendo tanto las capacidades de la fuerza laboral como las del capital industrial. La cifra indica que los daños ocasionados a la fuerza laboral constituyen la principal fuente de interrupciones de la producción en los primeros cinco periodos.

La Figura 2.c muestra la dinámica de la demanda final después del desastre. La línea verde indica el proceso de adaptación y recuperación de la demanda final. Esta variable incluye el comportamiento adaptado de los consumidores finales y la demanda de reconstrucción. Por otro lado, la línea roja muestra cuánto de esa demanda adaptada puede ser suministrada por la capacidad limitada de producción. Parte de la demanda que no puede ser satisfecha por la producción interna se suministra a través de las importaciones, como ilustra la línea negra. 


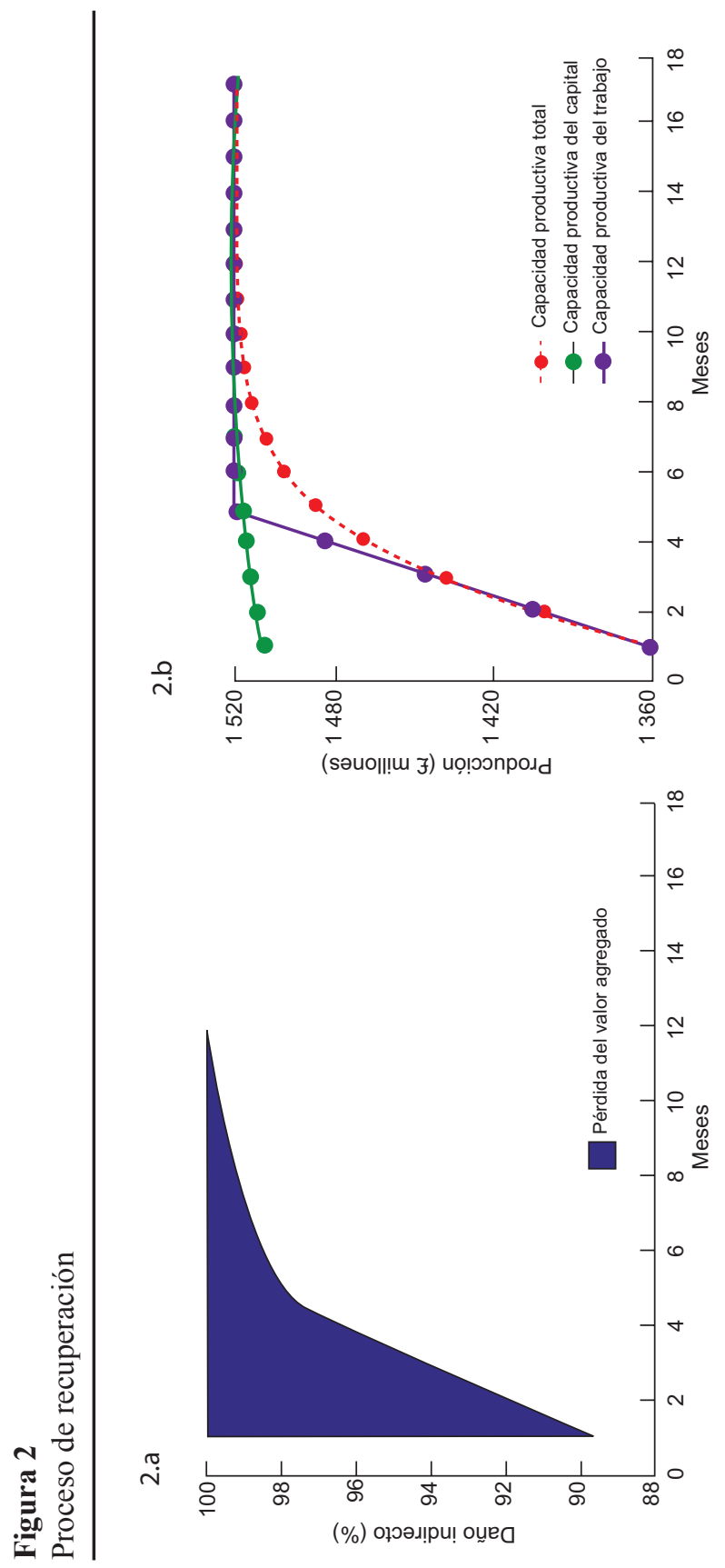



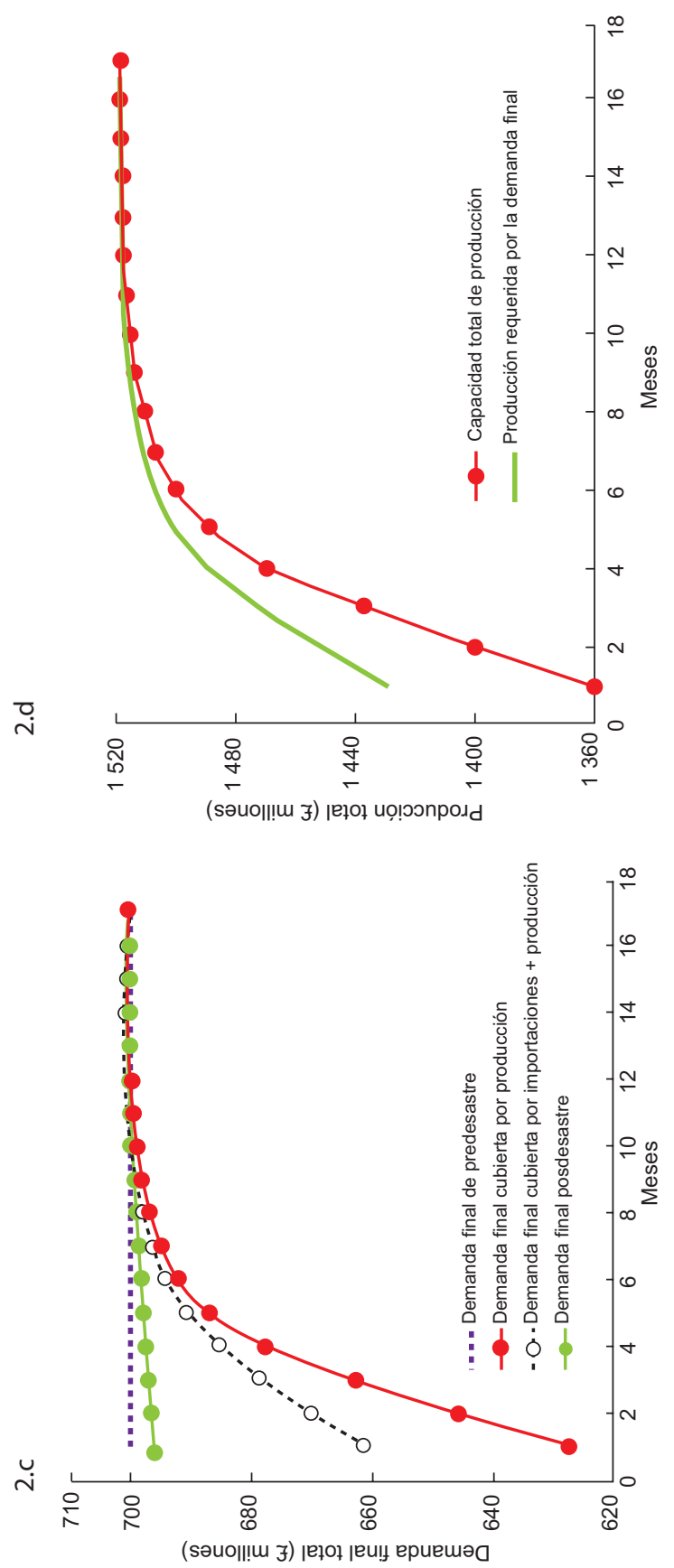
Finalmente, la Figura 2.d indica las desigualdades generadas durante el proceso de recuperación entre el nivel de producción requerido por la demanda final y la capacidad de producción que sobrevive a lo largo de las secuelas.

\subsection{Análisis sectorial}

Dado que este modelo parte de un modelo de IP, se pueden hacer análisis a nivel de sector industrial. Esto es especialmente útil para analizar la distribución de los efectos del choque a la economía, y que se propagan a lo largo de los sectores productivos, e incluso hacia otros sistemas económicos. Esta característica de la metodología se vuelve muy conveniente para la planificación en la gestión de riesgo de inundaciones y las políticas de adaptación.

La Figura 3 muestra la distribución del impacto económico, dividido por daños directos e indirectos, agregados en diez grupos industriales. Las proporciones de pérdidas directas e indirectas presentan una alta heterogeneidad entre los grupos. Por ejemplo, el sector de servicios públicos es el más afectado, con una pérdida directa que casi triplica la pérdida indirecta para el mismo grupo industrial. El total de daños en este grupo representa el $28 \%$ del costo económico total del desastre natural. Por el contrario, la composición de los daños en el sector financiero y profesional guarda una relación inversa. Es el sector con mayores costos indirectos, acumulando $23 \%$ de los daños indirectos totales. Por otro lado, sólo 5\% de los daños directos totales se concentraron en este grupo.

A un nivel más desagregado (de 46 sectores), la Figura 4 muestra los diez sectores más afectados por pérdidas económicas, tanto directas (a) como indirectas (b), respectivamente. Las pérdidas directas en el sector de electricidad y gas, por ejemplo, representan el $70 \%$ de las pérdidas económicas directas en el sector de servicios públicos y el $26 \%$ del total de los daños directos.

Cabe señalar que tres de las industrias aparecen en ambas categorías. Se trata de los sectores de electricidad y gas, almacenamiento y servicio postal, y metales. En conjunto, estos tres sectores concentran el $40 \%$ de los daños directos totales, el $12 \%$ de la pérdida indirecta total, y el $28 \%$ del impacto económico total de la inundación. Obsérvese que la escala es diferente en cada gráfica de la Figura 4, lo que implica una distribución más uniforme del daño indirecto, mientras que el daño directo está altamente concentrado en pocos sectores. 


\section{Figura 3}

Distribución sectorial del impacto económico

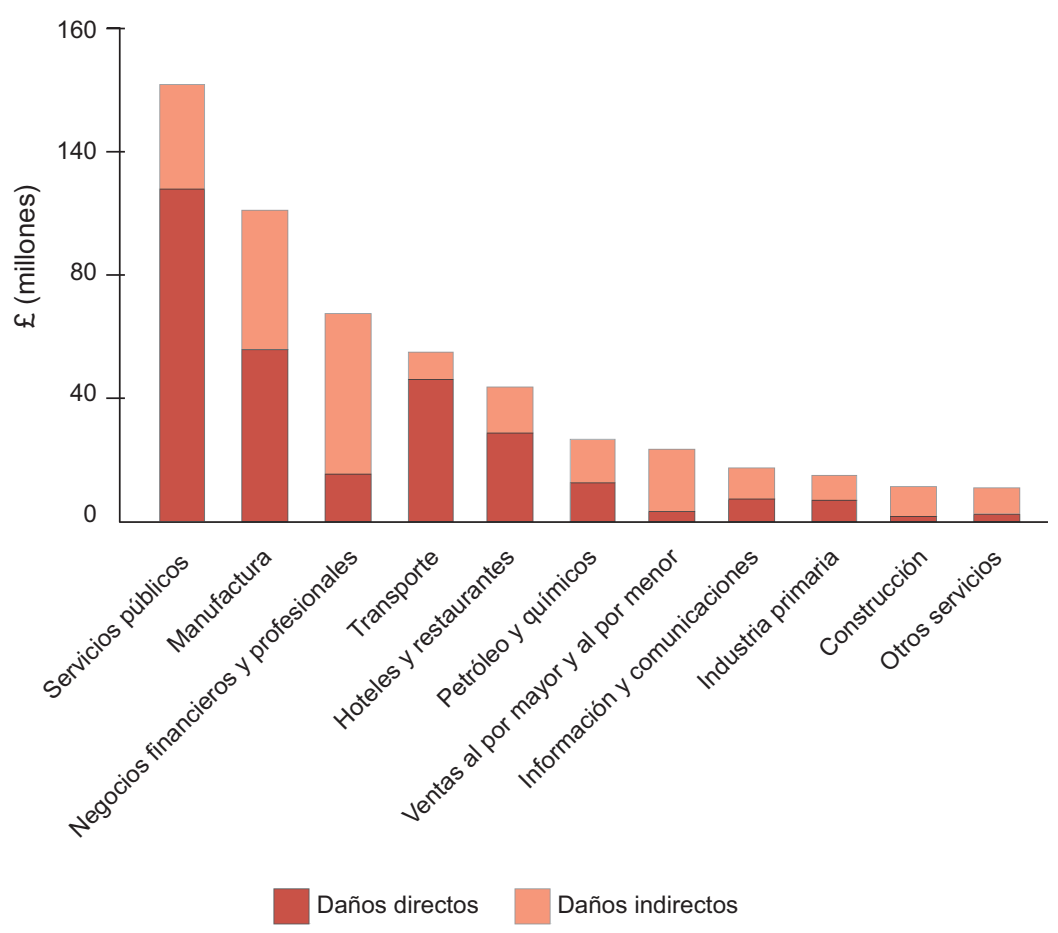

Fuente: Elaboración propia.

\subsection{Análisis comparado}

En un ejercicio comparativo, basado en los resultados de Mendoza-Tinoco et al. (2017), el modelo estima una recuperación más lenta para la ciudad de Sheffield (17 meses) que para la de Y\&H (14 meses). La diferencia puede deberse a que el análisis más detallado a nivel de ciudad permita capturar los efectos de los daños estructurales que sufrió la ciudad -la más afectada de la región-, mientras que éstos podrían difuminarse en los datos económicos de una zona más amplia.

Por otro lado, es notable el hecho de que, mientras en la región de $\mathrm{Y} \& \mathrm{H}$ los costos indirectos representan el 57\% del total, en la ciudad de Sheffield 


\section{Figura 4}

Sectores más afectados, por categoría de daños: directos e indirectos

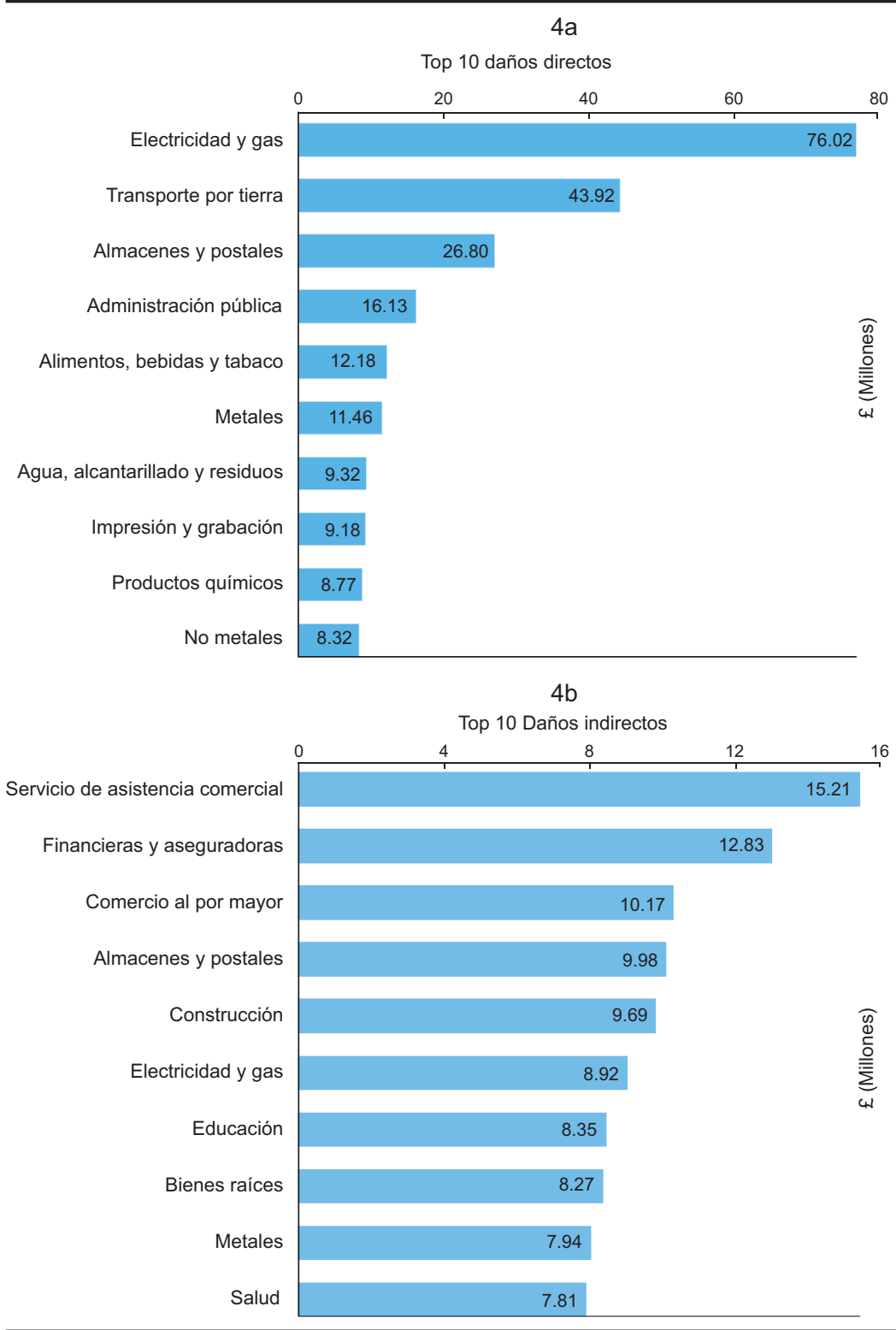

Fuente: Elaboración propia.

Estudios Demográficos y Urbanos, vol. 33, núm. 3 (99), 2018, pp. 699-732

ISSN 0186-7210; e ISSN 2448-6515; doi: http://dx.doi.org/10.24201/edu.v33i3.1786 
éstos sólo representan el 43\%. El Cuadro 1 muestra la distribución de los daños en cada región, por categoría. Esto muestra que las perturbaciones a la economía, por los daños materiales, se propagaron en mayor medida en la región de Y\&H que en la ciudad de Sheffield.

Finalmente, en una comparación por sector, las industrias que mayores daños materiales (o directos) sufrieron en Y\&H fueron las relacionadas con los servicios y provisión de agua, mientras que en Sheffield fueron los sectores de electricidad y gas. En ambos casos se trata de daños a la infraestructura. Mientras que en los costos indirectos los sectores más afectados en la región de Y\&H fueron el de tecnologías de la información y el comercio al por mayor, en la ciudad de Sheffield fueron los servicios de apoyo a los comercios, y los servicios financieros y de aseguradoras.

Los resultados del análisis ofrecen una simulación de la recuperación de la economía de Sheffield, dados los supuestos y datos considerados para la modelación. Puesto que es un evento pasado, resultaría de gran utilidad conocer los costos verdaderos de la inundación. Sin embargo, existen muy pocos reportes oficiales que consideren los costos indirectos ocasionados por restricciones en los factores productivos. El reporte de Ash et al. (2008) es de los pocos que ofrecen un panorama de las afectaciones a la producción de las empresas inducidos por los daños materiales. Ellos estiman que la afectación a la región de Sheffield por concepto de "pérdida de negocios y reducción de la productividad" es de $£ 92$ millones, lo cual es menos de la mitad de lo estimado con la metodología propuesta en este artículo. La estimación de daños indirectos en este reporte es mediante un modelo de insumo-producto estándar, el cual sólo considera los daños al capital industrial, y no al trabajo. Y sólo toma en cuenta el periodo inmediato después del desastre.

\section{Cuadro 1}

Comparación de daños por categoría con la región de Y\&H

\begin{tabular}{lcc}
\hline & $\begin{array}{c}\text { Yorkshire and The } \\
\text { Humber } \\
\text { (\%) }\end{array}$ & $\begin{array}{c}\text { Ciudad } \\
\text { de Sheffield } \\
\text { (\%) }\end{array}$ \\
\hline Costos directos residenciales & 12 & 2 \\
Costos directos industriales & 31 & 55 \\
Costos indirectos & 57 & 43 \\
\hline
\end{tabular}




\section{Conclusiones}

Los altos costos ocasionados por los desastres naturales requieren un análisis más comprehensivo para proveer de información más precisa y detallada sobre los daños para una eficaz implementación de las medidas de gestión de riesgos de desastres naturales. Esto coadyuvaría a mejorar las políticas de adaptación para la sostenibilidad económica de las ciudades afectadas. Por ejemplo, las inundaciones del verano de 2007 causaron una emergencia nacional en Inglaterra, siendo la ciudad de Sheffield la más afectada.

Este trabajo contribuye con el análisis empírico de un método para la estimación de los costos económicos totales causados por una inundación en la escala regional de una ciudad. Es importante la aplicación a esta escala porque permite la comparación con estudios previos en una región más amplia, lo que permite avanzar en la adaptabilidad de la metodología para otros casos de estudio. Asimismo, se incorpora a la metodología el concepto de matriz de capital, lo cual proporciona una mayor consistencia metodológica en el análisis de la recuperación económica, y permite identificar de manera más precisa los sectores involucrados en el proceso de recuperación y la dinámica que siguen a lo largo de la misma.

Este artículo es pionero en la aplicación de una metodología que considera diversos aspectos que perturbaron el equilibrio económico después de las inundaciones de 2007 en la ciudad de Sheffield. El análisis respalda la importante lección de que las pérdidas derivadas de un desastre son exacerbadas por los mecanismos económicos, y que los efectos indirectos (o daños indirectos) constituyen una proporción sustancial de los costos totales. También se observó que algunos de los sectores más afectados pueden ser aquellos que no son directamente dañados por las inundaciones. Para este estudio de caso, la proporción de daños indirectos representa más de la mitad del impacto económico total.

Esta investigación proporciona una evidencia cuantitativa para una mejor asignación de recursos para la gestión de riesgos por desastres naturales, en el que se encuentra que cualquier daño directo puede tener un impacto indirecto significativo a lo largo de la cadena de suministro económico. Las políticas de gestión de riesgos deberían considerar la minimización del impacto indirecto, como es el caso de los daños en sectores que son vulnerables a la pérdida de mano de obra, como los sectores de servicios. El no considerar los efectos indirectos induciría a errores en las acciones para la gestión de riesgos de desastres naturales, y conduciría a un uso ineficiente de los recursos destinados a ello. 
Hay, sin embargo, algunas advertencias que deben ser observadas. El presente estudio está sujeto a cierto grado de incertidumbre. Primero, la escasez de datos representa la principal fuente de incertidumbre, por lo que el uso de supuestos fuertes es inevitable en ciertos casos y se dificulta la aplicación del modelo a una gama más extensa de casos. Es debido a que existe una línea de investigación más desarrollada en el análisis de impacto de inundaciones que es más factible aplicarlo a este tipo de desastres naturales. En el caso específico de las inundaciones, el desarrollo de modelos de inundación y el uso de técnicas, como los sistemas geográficos de información, han evolucionado rápidamente en los últimos años, proporcionando nuevas fuentes de datos con gran precisión. También se ha avanzado en la construcción de las llamadas funciones de daños, lo que permitirá un análisis más preciso y más rápido en un futuro cercano. En segundo lugar, si bien el modelo responde eficazmente a los efectos indirectos en la economía regional afectada, la interconexión económica mundial nos obliga a llevar el análisis hacia un enfoque multirregional, si queremos realizar una evaluación de impacto exhaustiva. Por último, la investigación adicional sobre los efectos de los desastres sobre la fuerza laboral y el consumo mejoraría significativamente el análisis, ya que éstas son áreas que han atraído menos la atención de los investigadores.

Finalmente, la metodología podría representar significativos avances en el entendimiento de la dinámica de los daños económicos ocasionados por los desastres naturales que afectan a la economía mexicana. Es, sin embargo, necesario mencionar que esto impone retos importantes en la aplicación de la metodología, principalmente los relacionados con la estimación de daños directos y con la regionalización de los datos económicos. 


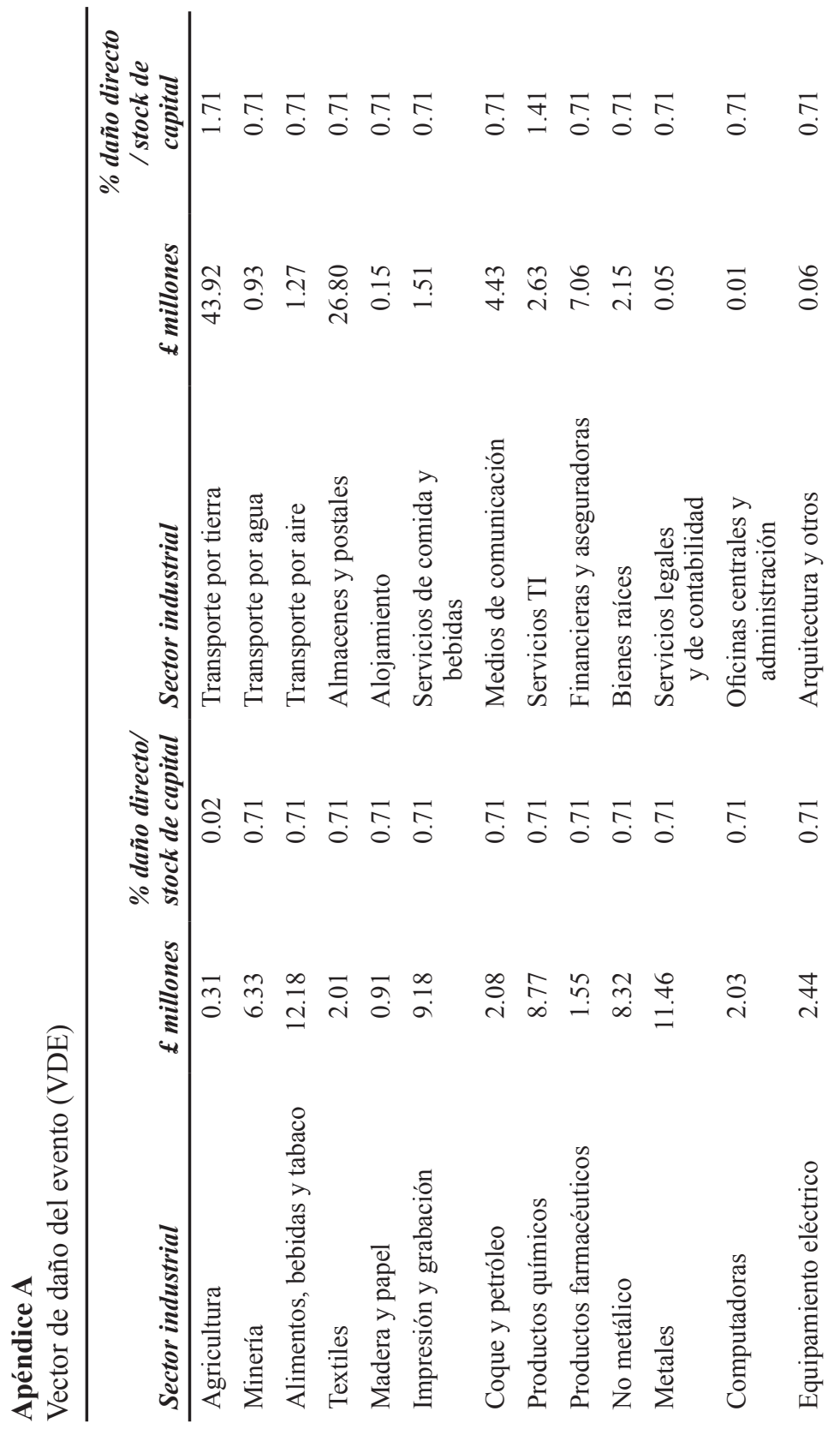




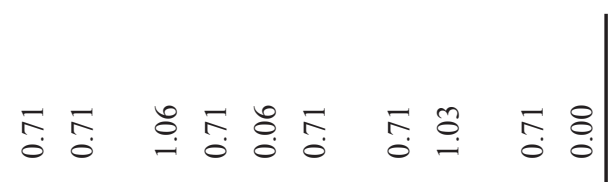

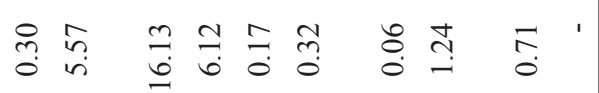

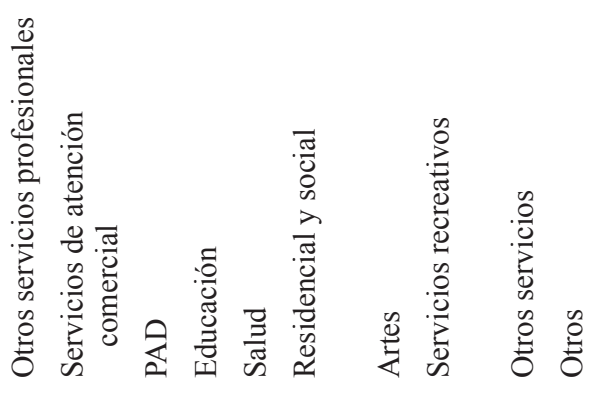

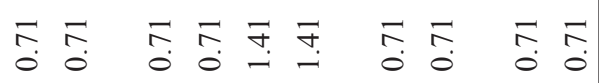

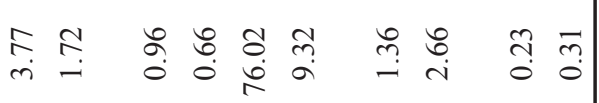

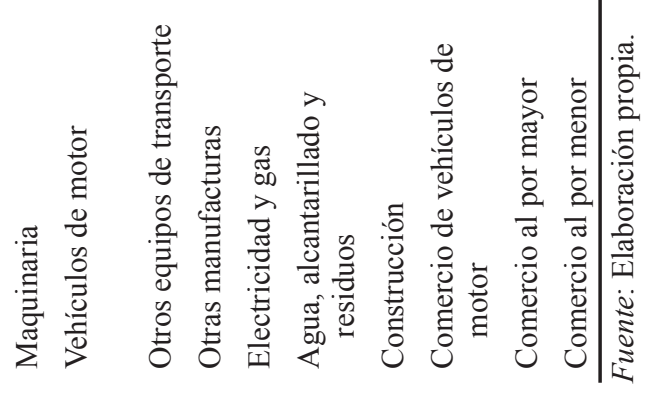




\section{Apéndice B}

\section{Regionalización de coeficientes técnicos de insumo-producto. Cocientes de localización aumentados de Flegg (AFLQ, por sus siglas en inglés)}

Se han desarrollado diversas técnicas dentro del campo de investigación del modelo de IP para regionalizar los coeficientes técnicos, siendo las técnicas estadísticas las más utilizadas. Este campo de investigación es conocido como análisis de insumo-producto regional y ha contado con las importantes contribuciones de diversos investigadores, como Richardson y Hewings, así como Flegg y Webber (Miller y Blair, 2009).

Para obtener la matriz regional de coeficientes técnicos para la ciudad de Sheffield se utilizó en este trabajo la técnica de los cocientes de localización aumentados de Flegg (Flegg y Webber, 2000; Miller y Blair, 2009; Romero, Mastronardi y Faye, 2012). Esta técnica busca corregir los coeficientes técnicos nacionales para representar la tecnología regional, dada la estructura económica regional.

Para ello se utilizan datos económicos sobre la economía local para escalar los coeficientes nacionales. Se utilizan principalmente datos sobre el empleo, ya que ésta es una de las fuentes de datos más confiables y disponibles a nivel subnacional. El proceso consiste en ajustar los coeficientes nacionales a la escala regional, midiendo el tamaño relativo de cada industria en la economía regional, en relación con el tamaño relativo de la misma industria para la economía nacional. Algunos parámetros también se ajustan para considerar el tráfico comercial entre la economía regional y otras regiones, y la posible especialización de una industria dentro de la región.

Entonces, el coeficiente técnico regional, $r_{i j}$, se deriva de los coeficientes técnicos nacionales, $a_{i j}$, cuando se vuelve a clasificar según un parámetro de economía regional o cociente de localización, $l q_{i j}$, tal como se muestra en la Ecuación 1.B:

$$
r_{i j}=l q_{i j} * a_{i j}
$$

donde $r_{i j}$ es la cantidad de insumos de la industria $i$ necesaria para producir una unidad de producto en la industria $j$. Aquí aplicamos uno de los cocientes de localización más utilizados, $l q_{i j}$, el AFLQ. El $l q_{i j}$ representa el cociente de localización que está alterando el coeficiente técnico nacional $\left(a_{i j}\right)$, para representar la tecnología regional. Para este caso, el término $l q_{i j}$ toma los valores de $\operatorname{los} A F L Q_{i j}$ descritos a continuación.

Partimos de los llamados cocientes de localización simples (SQL, por sus siglas en inglés) para evaluar la importancia relativa de cada industria 
regional $i$, tal como se describe en la Ecuación 2.B.

$$
s l q_{i}=\frac{r e_{i} / \text { tre }}{n e_{i} / \text { tne }} \equiv \frac{r e_{i}}{n e_{i}} * \frac{\text { tne }}{\text { tre }}
$$

donde tre es el empleo total en la región, the es el empleo total en el país, $r e_{i}$ es el empleo en la región que suministra el insumo, y $n e_{i}$ representa el empleo nacional en el mismo sector.

A continuación, el cociente de localización (LQ, por sus siglas en inglés) industrial cruzado (CILQ) se deriva del SQL para evaluar la importancia relativa de una industria proveedora $i$ con respecto a la industria compradora $j$ (véase la Ecuación 3):

$$
c i l q_{i j}=\frac{s l q_{i}}{s l q_{j}} \equiv \frac{r e_{i} / n e_{i}}{r e_{j} / n e_{j}}
$$

Posteriormente, Flegg y Webber (1997) refinaron la regionalización del LQ de Flegg (FLQ) para corregir la subestimación persistente de las importaciones regionales en el CILQ, a través del parámetro $\square=\left[\log _{2}(1+\right.$ tre $/$ tne) $]$ para obtener el FLQ. El parámetro $\square$ da flexibilidad a la fórmula, aunque su estimación está más relacionada con el análisis empírico. La fórmula establece que cuanto menor sea el valor de $\square$, mayor será el valor de $\square$, para cualquier relación dada (tre / the) (Flegg \& Webber, 2000).

Por último, en los AFLQ (Ecuación 4.B) se agrega un último parámetro para cubrir la posibilidad de especialización regional en algunos sectores $\left[\log _{2}\left(1+s l q_{j}\right)\right]$ :

$$
a f l q_{i j} \equiv c i l q_{i j} * *\left[\log _{2}\left(1+s l q_{j}\right)\right]
$$

A continuación, se obtiene la matriz regional de cocientes técnicos, $A_{\text {reg, }}$, al multiplicar la matriz de coeficientes técnicos nacionales $(A)$ por el cociente de localización correspondiente, AFLQ:

$$
\mathbf{A}_{\text {reg }}=\mathbf{A F L Q . * A}
$$

Para cada coeficiente técnico nacional $\left(a_{i j}\right)$ obtenemos la contrapartida regional $\left(r_{i j}\right)$ :

$$
r_{i j}=a f l q_{i j} * a_{i j}
$$




\section{Bibliografía}

Ash, J., Fenn, T., Daly, E., y Wels, N. (2008). Economic impacts of flood risk on Yorkshire and Humber: Cost of 2007 floods. Inglaterra.

Bockarjova, M., Steenge, A., y van der Veen, A. (2004). On direct estimation of initial damage in the case of a major catastrophe: Derivation of the "basic equation”. Disaster Prevention and Management, 13(4), 330-336. Recuperado de http://www.emeraldinsight.com/doi/pdfplus/10.1108/09653560410556555

Burton, I., Challenger, B., Huq, S., Kein, R. J. T. y Yohe, G. (2001). Adaptation to climate change in the context of sustainable development and equity. En Intergovernmental Panel on Climate Change (ed.), Climate change 2001: Impacts, adaptation and vulnerability. Cambridge University Press.

Chen, S. Q., Fath, B. D. y Chen, B. (2011). Information-based network environ analysis: A system perspective for ecological risk assessment. Ecological Indicators, 11, 1664-1672. Recuperado de https://doi.org/10.1016/j.rser.2014.10.017

Chen S. Q., Chen, B. y Fath, B. D. (2015). Assessing the cumulative environmental impact of hydropower construction on river systems based on energy network model. Renewable \& Sustainable Energy Reviews, 42, 78-92. Recuperado de https://doi.org/10.1016/j.rser.2014.10.017

Cochrane, H. (1997). Economic impacts of a Midwestern earthquake. NCEER Bulletin, 11(1), 1-20. Recuperado de http://citeseerx.ist.psu.edu/viewdoc/download? doi $=10.1 .1 .652 .169 \&$ rep $=$ rep1\&type $=$ pdf

Cochrane, H. (2004). Economic loss: Myth and measurement. Disaster Prevention and Management, 13(4), 290-296. Recuperado de http://www.emeraldinsight. com/doi/pdfplus/10.1108/09653560410556500

Cole, S. (2003). Protection, risk, and disaster in economic network. En A. van der Veen, A. L. V. Arellano y J. P. Nordvik (eds.), Joint NEDIES and University of Twente Workshop in search of a common methodology on damage estimation. Delft: Office for Official Publications of the European Communities.

Committee on Climate Change (2016). UK Climate Change Risk Assessment 2017. Londres: UK Government. Recuperado de www.theccc.org.uk/uk-climatechange-risk-assessment-2017

Dietzenbacher, E. y Lahr, M. L. (2013). Expanding extractions. Economic Systems Research, 25(3). Recuperado de http://dx.doi.org/10.1080/09535314.201 3.774266

Dietzenbacher, E. y Miller, R. E. (2015). Reflections on the inoperability input-output model. Economic Systems Research, 27(4), 478-486. https://www.tandfonline. com/doi/full/10.1080/09535314.2015.1052375

Flegg, A. T. y Webber, C .D. (2000). Regional size, regional specialization and the FLQ formula. Regional Studies, 34(6), 563-569. Recuperado de http:/www. tandfonline.com/doi/pdf/10.1080/00343400050085675?needAccess=true

Greenberg, M. R., Lahr, M. y Mantell, N. (2007). Understanding the economic costs and benefits of catastrophes and their aftermath: A review and suggestions for 
the U.S. Federal Government. Risk Analysis, 27(1). Recuperado de http://on linelibrary.wiley.com/doi/10.1111/j.1539-6924.2006.00861.x/epdf

Haimes, Y. y Jiang, P. (2001). Leontief-based model of risk in complex interconnected infrastructures. Journal of Infrastructure Systems, 7(1), 1-12. Recuperado de https://ascelibrary.org/doi/abs/10.1061/\%28ASCE\%291076-0342\%282001 $\% 297 \% 3$ A $1 \% 281 \% 29$

Haimes, Y. Y., Asce, F., Horowitz, B. M., Lambert, J. H., Santos, J. R. y Crowther, K. G. (2005). Inoperability input-output model (IIM) for interdependent infrastructure sectors. I: Theory and methodology. Journal of Infrastructure Systems, 11(2), 67-79. Recuperado de https://ascelibrary.org/doi/abs/10.1061/\%28ASCE $\% 291076-0342 \% 282005 \% 2911 \% 3$ A2\%2867\%29

Hallegatte, S. (2008). An adaptive regional input-output model and its application to the assessment of the economic cost of Katrina. Risk Analysis, 28(3), 779-799. Recuperado de https://www.ncbi.nlm.nih.gov/pubmed/18643833

Hallegatte, S., y Przyluski, V. (2010). The economics of natural disasters. Concepts and methods (Policy Research Working Paper, núm. 5507). Washington: Banco Mundial.

Kajitani, Y. y Tatano, H. (2014). Estimation of production capacity loss rate after the great east Japan earthquake and tsunami in 2011. Economic System Research, 26(1). Recuperado de https://doi.org/10.1080/09535314.2013.872081

Koks, E. E., Bockarjova, M., de Moel, H. y Aerts, J. C. J. H. (2014). Integrated direct and indirect flood risk modeling: Development and sensitivity analysis. Risk Analysis, 35(5). Recuperado de https://doi.org/10.1111/risa.12300

Leung, M., Haimes, Y. Y. y Santos, J. R. (2007). Supply- and output-side extensions to the inoperability input-output model for interdependent infrastructures. Journal of Infrastructure Systems, 13(4), 299-310. Recuperado de https://ascelibrary. org/doi/abs/10.1061/\%28ASCE\%291076-0342\%282007\%2913\%3A4\% $28299 \% 29$

Li, J., Crawford-Brown, D., Syddall, M. y Guan, D. (2013). Modeling imbalanced economic recovery following a natural disaster using input-output analysis. Risk Analysis, 33(10). Recuperado de https://www.ncbi.nlm.nih.gov/pubmed/23614394

Mendoza-Tinoco, D., Guan, D., Zeng, Z., Xia, Y. y Serrano, A. (2017). Flood footprint of the 2007 floods in the UK: The case of the Yorkshire and The Humber Region. Journal of Cleaner Production, 168, 655-667 Recuperado de https://doi. org/10.1016/j.jclepro.2017.09.016

Messner, F., Penning-Rowsell, E., Green, C., Meyer, V., Tunstall, S. y van der Veen, A. (2007). Evaluating flood damages: guidance and recommendations on principles and methods (Reporte de investigación, núm. T09-06-01). FLOODsite Project. Recuperado de http://www.floodsite.net/html/partner_area/project_docs/ T09_06_01_Flood_damage_guidelines_D9_1_v2_2_p44.pdf

Miller, R. E. y Blair, P. D. (2009). Input output analysis: Foundations and extensions. Cambridge: US: Prentice-Hall.

Okuyama, Y. (2004). Modeling spatial economic impacts of an earthquake: Input-output 
approaches. Disaster Prevention and Management, 13(4), 297-306. Recuperado de http://www.emeraldinsight.com/doi/pdfplus/10.1108/09653560410556519

Okuyama, Y. (2007). Economic modeling for disaster impact analysis: Past, present, and future. Economic Systems Research, 19(2), 115-124. Recuperado de http:// www.tandfonline.com/doi/pdf/10.1080/09535310701328435?needAccess $=$ true

Okuyama, Y. (2009) Critical review of methodologies on disaster impact estimation. (Documento de trabajo). Assessment on the Economics of Disaster Risk Reduction, the Global Facility for Disaster Reduction and Recovery (GFDRR), World Bank.

Okuyama, Y. (2014). Disaster impact and input-output analysis. Economic Systems Research, 26(1), 1-12. Recuperado de https://doi.org/10.1080/09535314.2013. 871505

Oosterhaven, J. (2017). On the limited usability of the inoperability IO model. Economic Systems Research, 29(3), 452-461. Recuperado de https://doi.org/10.10 80/09535314.2017.1301395

Oosterhaven, J. y Bouwmeester, M. C. (2016). A new approach to modeling the impact of disruptive events. Journal of Regional Science, 56(4), 583-595. Recuperado de http://onlinelibrary.wiley.com/doi/10.1111/jors.12262/epdf

Pitt, M. (2008). The Pitt review. Londres. Recuperado de http://www.climatejust.org. uk/resources/pitt-review-2008

Romanoff, E. y Levine, S. (1981). Anticipatory and responsive sequential interindustry models. IEEE Transactions on Systems, Man, and Cybernetics, 11(3), 181186. Recuperado de http://ieeexplore.ieee.org/document/4308650/

Romero, C. A., Mastronardi, L. J. y Faye, M. J. (2012). Location quotient analysis. The case of Cordoba state. Munich Personal RePEc Archive (36997). Recuperado de https://mpra.ub.uni-muenchen.de/36997/e

Rose, A. Z. (1995). Input-output economics and computable general equilibrium models. Structural Change and Economic Dynamics, 6(3), 295-304. Recuperado de https://doi.org/10.1016/0954-349X(95)00018-I

Rose, A. Z. (2004). Economic principles, issues, and research priorities in hazard loss estimation. En Y. Okuyama y S. E. Chang (eds.), Modelling spatial and economic impacts of disasters. Berlín, Heidelberg y Nueva York: Springer-Verlag.

Santos, J. R. (2006). Inoperability input-output modeling of disruptions to interdependent economic systems. Systems Engineering, 9(1), 20-34. Recuperado de http://onlinelibrary.wiley.com/doi/10.1002/sys.20040/epdf

Santos, J. R. y Haimes, Y. Y. (2004). Modeling the demand reduction input-output (i-o) inoperability due to terrorism of interconnected infrastructures. Risk Analysis, 24(6), 1437-1451. Recuperado de https://www.ncbi.nlm.nih.gov/pubmed/ 15660602

Santos, J. R. y Rehman, A. (2012). Risk-based input-output analysis of hurricane impacts on interdependent regional workforce systems. Natural Hazards, 65(1), 391-405. Recuperado de https://ink.springer.com/article/10.1007/s11069-0120369-0 
Santos, J. R., Yu, S., Pagsuyoin, S. y Tan R. (2014). Time-varying disaster recovery model for interdependent economic systems using hybrid input-output and event tree analysis. Economic System Research, 26(1). Recuperado de doi: https://doi. org/10.1080/09535314.2013.872602

Steenge, A. E., y Bockarjova M. (2007). Thinking about rigidities and imbalances in post-catastrophe economies: An input-output based proposition. Economic Systems Research, 19(2), 205-223. Recuperado de https://research.vu.nl/en/ publications/thinking-about-rigidities-and-imbalances-in-post-catastrophe-econ

Triple E Consulting. (2014). Climate extremes. Defining a pilot approach on estimating the direct and indirect impacts on economic activity. European Commission.

Vanneuville W., Kellens, W., Maeyer, P. D., Reniers, G. y Witlox, F. (2011). Is “flood risk management" identical to "flood disaster management"? Earthzine, 8. Recuperado de https://earthzine.org/2011/03/21/is-flood-risk-managementidentical-to-flood-disaster-management/

Veen, A. V. d. (2004). Disasters and economic damage: macro, meso and micro approaches. Disaster Prevention and Management, 13(4), 274-279. Recuperado de http://www.emeraldinsight.com/doi/pdfplus/10.1108/09653560410556483

Veen, A. V. d. y Logtmeijer, C. (2003). How vulnerable are We for flooding? A GIS approach. En A. van der Veen, A. L. V. Arellano y J. P. Nordvik (eds.), In search of a common methodology for damage estimation. Delft: Office for Official Publications of the European Communities / Joint NEDIES / University of Twente.

Xu, W., Hong, L., He, L., Wang, S. y Chen, X. (2011). Supply-driven dynamic inoperability input -output price model for interdependent infrastructure systems. Journal of Infrastructure Systems, 17, 151-162. Recuperado de https://ascelibrary. org/doi/abs/10.1061/(ASCE)IS.1943-555X.0000058

\section{Acerca de los autores}

David Mendoza Tinoco es licenciado en Economía por la Universidad Nacional Autónoma de México, con medalla al mérito. Cursó la maestría en Economía en El Colegio de México, A. C., y tiene un doctorado en Desarrollo Internacional por la Universidad de Anglia del Este, en el Reino Unido. Actualmente se desempeña como investigador de proyecto en el Programa de Análisis Económico para México del Centro de Estudios Económicos de El Colegio de México, A. C. Anteriormente colaboró como investigador en la Unión Europea, para el proyecto BASE: Bottom-Up Climate Adaptation Strategies towards a Sustainable Europe. También colaboró como investigador en la Comisión Europea, para el proyecto Climate Extremes Project: Defining a pilot approach on estimating the direct and indirect impacts on economic activity. 


\begin{abstract}
Alba Verónica Méndez Delgado es licenciada en Economía por la Facultad de Economía de la Universidad Autónoma de Coahuila (UAdeC). Tiene una maestría en Economía Regional, con mención honorífica, por el Centro de Investigaciones Socioeconómicas (CISE) de la UAdeC. Es doctora en Economía por el Centro de Estudios Económicos de El Colegio de México, A. C. Actualmente se desempeña como profesora investigadora en el CISE, y realiza actividades de docencia en la Facultad de Economía y en el CISE de la UAdeC, en las materias de Macroeconomía III (crecimiento económico) y Econometría Espacial.
\end{abstract}

Fecha de recepción: 22 de agosto de 2017.

Fecha de aceptación: 20 de diciembre de 2017. 